\title{
Six-dimensional measurements of trains of high brightness electron bunches
}

\author{
A. Cianchi, ${ }^{1,}{ }^{*}$ D. Alesini, ${ }^{2}$ M. P. Anania, ${ }^{2}$ A. Bacci, ${ }^{3}$ M. Bellaveglia, ${ }^{2}$ M. Castellano, ${ }^{2}$ \\ E. Chiadroni, ${ }^{2}$ D. Di Giovenale, ${ }^{2}$ G. P. Di Pirro, ${ }^{2}$ M. Ferrario, ${ }^{2}$ A. Gallo, ${ }^{2}$ L. Innocenti, ${ }^{4}$ \\ A. Mostacci, ${ }^{5}$ R. Pompili, ${ }^{2}$ A. R. Rossi, ${ }^{3}$ J. Scifo, ${ }^{2}$ V. Shpakov, ${ }^{2}$ C. Vaccarezza, ${ }^{2}$ and F. Villa ${ }^{2}$ \\ ${ }^{1}$ INFN-Roma Tor Vergata and University of Rome "Tor Vergata", \\ Via della Ricerca Scientifica, 1-00133 Rome, Italy \\ ${ }^{2}$ INFN-LNF, Via E. Fermi, 40-00044 Frascati, Rome, Italy \\ ${ }^{3}$ INFN-Sezione di Milano, via Celoria 16, 20133 Milano, Italy \\ ${ }^{4}$ University of Rome "Tor Vergata", Via della Ricerca Scientifica, 1-00133 Rome, Italy \\ ${ }^{5}$ University La Sapienza of Roma, via Antonio Scarpa 24, 00133 Roma, Italy
}

(Received 10 April 2015; published 31 August 2015)

\begin{abstract}
Trains of ultrashort electron pulses with $\mathrm{THz}$ repetition rate, so-called comblike beams, are assuming an ever growing interest in plasma-based acceleration. In particle-driven plasma wakefield acceleration (PWFA), a train of driver bunches with separation of the order of plasma wavelength, i.e., $300 \mu \mathrm{m}$, resonantly excites a plasma wake, which accelerates a trailing witness bunch, injected at the accelerating phase. Comblike beams have great potentialities in different fields of applications. In particular, radiation sources, such as free-electron lasers and $\mathrm{THz}$ radiation, take advantage from the possibility to tailor electron beams modulated both in time and energy, to customize emission bandwidth and temporal properties. In these scenarios, the manipulation of longitudinal phase space to investigate different bunch configurations, in terms of energy and time separation, is founded on the knowledge of the 6D phase space of each bunch in the train. In this paper we present the methods developed at the SPARC_LAB test facility in order to fulfill the requirements. Starting from conventional diagnostics, therefore applying well-known tools using more than one diagnostic at the same time, we have completely characterized not only the full 6D phase space of a comblike electron beam with $\mathrm{THz}$ repetition rate, but also each single bunch within the train. To our knowledge, this is the first time such a measurement has been performed. Experimental results for multibunch trains in different configurations, suitable for PWFA applications, will be shown and discussed.
\end{abstract}

DOI: 10.1103/PhysRevSTAB.18.082804

PACS numbers: 29.27.-a, 41.85.-p, 41.75.Fr

\section{INTRODUCTION}

A comblike beam is a train of short electron pulses, in the order of hundreds of femtoseconds or less with picosecond time separation. The generation of such a beam relies on different schemes, for instance a single long bunch is sliced by a mechanical slits system placed in a dispersive area [1] or a train of bunches is longitudinally manipulated using velocity bunching [2]. The first system has the advantage of an easy implementation, being necessary however a dispersive arm. However it suffers for a limited flexibility because both bunch length and time separation can only be changed by changing the slit system. The second one is much more flexible, being possible to modify the beam properties playing with the longitudinal phase space, with laser pulse shaping and the rf accelerating phases. However being a dynamical setup it is more demanding in terms of $\mathrm{rf}$ stability with respect to the other one.

*alessandro.cianchi@roma2.infn.it

Published by the American Physical Society under the terms of the Creative Commons Attribution 3.0 License. Further distribution of this work must maintain attribution to the author(s) and the published article's title, journal citation, and DOI.
So far one of the main applications of comblike beams is the production of two color free-electron laser (FEL) radiation $[3,4]$ using a pulse train with bunches at different energy. In one case, an electron beam with two energy level distribution, quasioverlapped in time, produces a train of femtosecond FEL pulses emitting at two frequencies. On the other hand, the two bunches train separated both in time and energy will give rise to two color FEL radiation pulses with a given time delay, to be used for pump and probe experiments. Due to the different matching conditions of the transverse phase spaces inside the undulator, the knowledge of transverse and longitudinal single bunch parameter is mandatory. In order to have a balanced energy gain for both FEL pulses, matching conditions should be satisfied by both electron bunches.

Another application of comblike electron beams concerns $\mathrm{THz}$ radiation. $\mathrm{THz}$ sources are assuming growing importance mainly due to their nonionizing properties. Linac driven sources offer high power pulses but reduced tunability. $\mathrm{THz}$ sources driven by comblike beam have been recently developed $[5,6]$ in order to provide tunable, narrowband, few-cycle, and multicycle coherent $\mathrm{THz}$ radiation. A precise control of the bunch temporal spacing is thus mandatory to tune the source wavelength. 
Last, but not least, one of the most challenging applications of such beams concerns their use in the development of particle-driven plasma wakefield acceleration (PWFA). PWFA has recently [7] demonstrated its feasibility to accelerate electron beams. The main goal is to deliver a train of driver bunches that resonantly excite a plasma wake, transferring energy to the plasma. A witness bunch, injected at a distance corresponding to half integer of the plasma wavelength from the last driver, will gain energy from plasma. Using this scheme it is in principle possible to achieve very high gradient, higher than $1 \mathrm{GV} / \mathrm{m}$ and up to $100 \mathrm{GV} / \mathrm{m}$. In order to deliver a useful beam, the energy spread and the transverse emittance must be kept under control. In this scenario the knowledge of the longitudinal and transverse characteristics of the single bunches are of paramount importance in order to match the transverse size at the beginning of the plasma channel and to set the proper distance of the bunches to tune the plasma oscillation.

Being the applications mainly linac oriented, single passage machine, intercepting diagnostics are allowed. Due to the high repetition rate of the bunches, intensified cameras cannot be used to collect and discriminate each bunch in the train, being not fast enough (nanosecond scale) for $\mathrm{THz}$ scale repetition rate. The only possibility is to separate the beams and to image them at the same time. A dipole can divide them when they are separated in energy, while a RFD ( $r f$ deflector) allows one to distinguish between them when the separation is in time. We have used both devices for our measurements.

Nevertheless, a nonintercepting device, such as the one based on electro-optic sampling, can be also used to characterize the longitudinal bunch size and shape. Details can be found in Ref. [8].

\section{EXPERIMENTAL SETUP}

The experiments presented in the paper have been conducted at the SPARC_LAB test facility [9], characterized by a high brightness photoinjector, able to deliver electron beams as short as few tens of femtoseconds, with $1 \mathrm{~mm}$-mrad transverse projected normalized emittance and

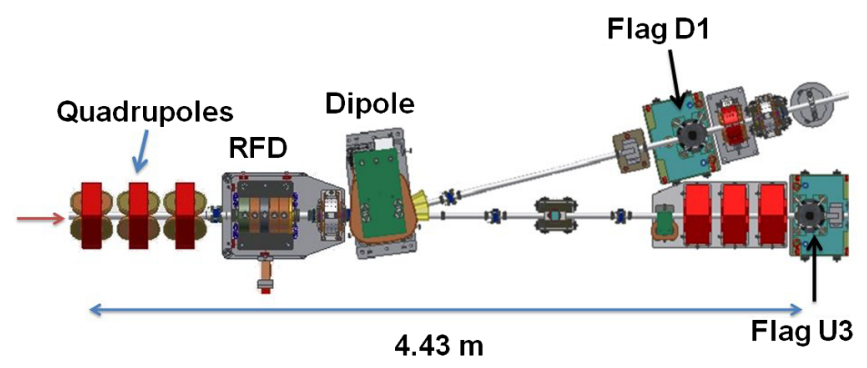

FIG. 1. Sketch of the SPARC_LAB diagnostic section beam line. The beam is coming from the left side. The drawing is not to scale. From left to right are visible the quadrupole triplet, the RFD (rf deflector), the dipole and the two view screens used in the measurement (named as flag D1 and U3). energy up to $180 \mathrm{MeV}$. The diagnostic section transfer line is sketched in Fig. 1.

Three quadrupoles are followed by the RFD and a dipole. Our RFD [10] has a vertical deflecting mode, so the longitudinal property of the beam is imaged on the transverse vertical plane. Two view screens are placed downstream, one on the straight line (U3), the other on a 14 degrees bending arm (D1). The distance between the screens and the dipole center is $2642 \mathrm{~mm}$ for U3 and $3274 \mathrm{~mm}$ for D1. Both of them are composed of a Yag: Ce crystal, normal to the beam line, $100 \mu \mathrm{m}$ thick, with a 45 degrees mirror placed on the back to eliminate depth of field effect [11]. The light is then collected by a $50 \mathrm{~mm} \mathrm{~F} 2.8$ lens and recorded with a Basler scout A640 CCD camera.

\section{LONGITUDINAL MEASUREMENT}

The simultaneous use of RFD and dipole magnet allows a single shot longitudinal phase space measurement. One of the big advantages of using a RFD is the self-calibrating property. The change of a known quantity, the phase of $\mathrm{rf}$ deflecting field, reflects in moving the beam in the vertical axis. Equating the number of the pixels with the phase difference gives the calibration ratio between dimension and phase. The vertical dimension on the screen reflects the bunch length, and the time separation in a comblike beam. The resolution of such a device is limited by one of the following effects: resolution of the optical system, deflecting voltage and beam emittance. In our case the main limitation comes from the beam emittance. In such a measurement the dimension on the vertical axis is given by [12] $\sigma_{y}=\sqrt{\sigma_{y_{-} \mathrm{RFD}}^{2}+\sigma_{y_{-} \text {beam }}^{2}}$ being $\sigma_{y}$ the measured quantity, $\sigma_{y_{-} \text {RFD }}$ the elongation due to the RFD and $\sigma_{y_{-} \text {beam }}$ the dimension on the screen without RFD, i.e., $\sigma_{y \_ \text {beam }}=$ $\sqrt{\varepsilon_{y} \beta_{y_{-} \text {screen }}}$ where $\varepsilon_{y}$ is the vertical emittance and $\beta_{y_{-} \text {screen }}$ is the Twiss function at the screen position. If the first term in the square root is equal or less to the second, the effect of the RFD is negligible. With $2 \mathrm{MW}$ power available for our $\mathrm{RFD}$, a camera pixel size equal to $10 \mu \mathrm{m}$, imaged with a $1: 3$ magnification, the intrinsic limit of resolution is about $18 \mathrm{fs}$ at $100 \mathrm{MeV}$ and $24 \mathrm{fs}$ at $160 \mathrm{MeV}$ for instance. But the dimension of the spot size without RFD, i.e., $\sigma_{y_{-} \text {beam }}$, basically doubles these values.

The resolution in energy depends on the optical magnification of the view screen, and the magnetic field in the dipole. With an equivalent pixel size of about $30 \mu \mathrm{m}$ on our camera, we have about $5 \mathrm{keV}$ resolution at $110 \mathrm{MeV}$ and $7 \mathrm{keV}$ at $160 \mathrm{MeV}$. But when the dipole is used with the RFD in order to obtain the longitudinal phase space, also the induced energy spread from the deflector must be taken into account. We have already evaluated and measured this effect in a former paper [13], being less than $20 \mathrm{keV}$.

One of the working points under studying at SPARC_LAB concerns the manipulation of a train of three 

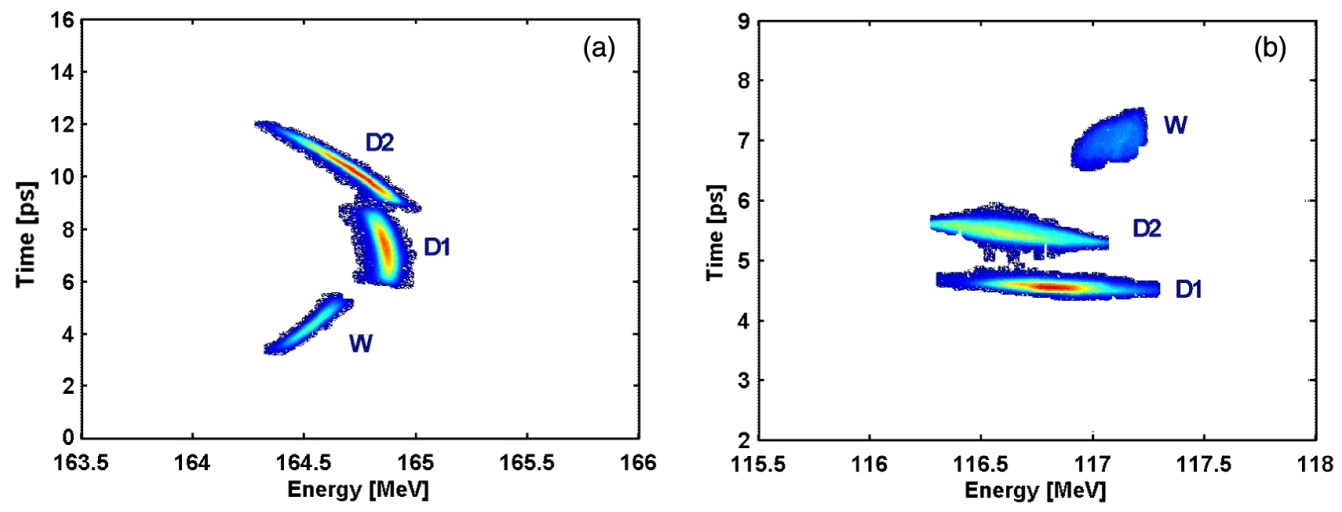

FIG. 2. (a) Measured longitudinal phase space with a comblike beam on crest. (b) Same structure at the overcompression phase which allows the bunches to be separated in time. The witness beam is indicated with $W$ and the drivers with D1 and D2.

bunches to be used for particle-driven resonant plasma wakefield acceleration, as shown in Fig. 2.

Two driver beams contain $44 \%$ (D1) and $42 \%$ (D2) of the total charge $(100 \mathrm{pC})$ respectively, while the witness beam has about $14 \%$. The charge distribution is set in order to have more charge in the drivers with respect to the witness. Knowing from a toroid the total value of the charge, the relative amount for every bunch is computed weighting the overall intensity of every single beamlet image. The phase space indicated with letter A shows the three bunches in on crest operation, i.e., all three traveling accelerating sections are at the rf field phase of maximum energy. The results are summarized in Table I. In parentheses are indicated the errors. When the statistical error is negligible with respect to the sampling dimension, we have reported this value.

Using the velocity bunching technique [14] and adjusting the phase of the other sections to partially compensate the induced energy spread it is possible to achieve a train of very short bunches and reduced energy spread, as shown in Fig. 2, phase space B. The resulting parameters are reported in Table II. With RFD and a dipole it is possible to measure in a single shot all the phase space longitudinal parameters of such a bunch train, including the relative distance between them and their individual charge.

\section{EMITTANCE MEASUREMENT}

Emittance measurements have been performed using the well-known quadrupole scan technique [15]. Different strategies must be applied depending on different scenarios: either trains with pulses almost overlapped in time, but not in energy or with same energy but separated in time.

\section{A. Bunches mainly separated in energy}

For a train of pulses separated in energy but not in time we need a dispersive element, the dipole and the flag D1. The emittance measurement is done taking into account the dipole contribution. We separate the problem for the vertical and the horizontal plane. In the vertical plane, using the notation of the $\sigma$-matrix [16], a matrix containing the Twiss parameters,

$$
\sigma_{33}=R_{33}^{2} \sigma_{033}+2 R_{33} R_{34} \sigma_{034}+R_{34}^{2} \sigma_{044}
$$

where $\sigma_{33}=\sqrt{\varepsilon_{y} \beta_{y}}$ is the beam vertical dimension at the measurement position, $\sigma_{0 i j}$ the element of the $\sigma$-matrix in the position where we want to know the value of the Twiss parameters and $R_{i j}$ the element of the $R$ transport matrix, which links the final and the initial $\sigma$-matrix elements as

$$
\sigma_{\text {final }}=R \sigma_{\text {start }} R^{T}
$$

Here and after we consider only the first order optics, because even in compression regime the energy spread of the single bunch is not so large $(\leq 0.3 \%)$ to make appreciable the contribution of high order terms. In the vertical plane there is no term coming from dispersion but, using a rectangular dipole, we must take into account in the transport matrix the vertical focusing according to [16].

To validate the transport matrix we measured the vertical emittance with a single bunch on both screens, D1 and U3. We usually use only one quadrupole for such a scan in order to minimize the chromatics effects of the magnetic elements and their effects on the emittance [17]. The comparison

TABLE I. Beam parameter for longitudinal phase space A in Fig. 2.

\begin{tabular}{lccccc}
\hline \hline Bunch & Beam energy (MeV) & Energy spread (\%) & Position (ps) & Bunch length (ps) & Longitudinal emittance $(\mathrm{keV} * \mathrm{~mm})$ \\
\hline$W$ & $164.50(0.02)$ & $0.051(0.013)$ & $4.28(0.02)$ & $0.54(0.02)$ & $5.3(0.2)$ \\
D1 & $164.82(0.02)$ & $0.030(0.013)$ & $7.12(0.02)$ & $0.73(0.02)$ & $9.8(0.3)$ \\
D2 & $164.66(0.02)$ & $0.086(0.013)$ & $10.01(0.02)$ & $0.74(0.02)$ & $9.6(0.5)$ \\
Whole & $164.71(0.02)$ & $0.092(0.013)$ & $7.91(0.02)$ & $2.13(0.02)$ & $96.5(1.4)$ \\
\hline \hline
\end{tabular}


TABLE II. Beam parameter for longitudinal phase space B in Fig. 2.

\begin{tabular}{lccccc}
\hline \hline Bunch & Beam energy $(\mathrm{MeV})$ & Energy spread $(\%)$ & Position $(\mathrm{ps})$ & Bunch length $(\mathrm{ps})$ & Longitudinal emittance $(\mathrm{keV} * \mathrm{~mm})$ \\
\hline$W$ & $117.12(0.02)$ & $0.067(0.002)$ & $6.39(0.02)$ & $0.190(0.03)$ & $4.0(0.2)$ \\
D1 & $116.65(0.02)$ & $0.158(0.005)$ & $5.02(0.02)$ & $0.12(0.02)$ & $5.6(0.2)$ \\
D2 & $116.82(0.02)$ & $0.175(0.009)$ & $4.19(0.02)$ & $0.08(0.02)$ & $4.6(0.4)$ \\
Whole & $116.80(0.02)$ & $0.210(0.005)$ & $4.92(0.02)$ & $7.67(0.02)$ & $53.4(1.2)$ \\
\hline \hline
\end{tabular}

between the measurements on screens $\mathrm{U} 3$ and D1 is reported in Table III.

In the single pulse run, images from the CCD are filtered to remove background noise and calculate the second momenta of the intensity distribution [18]. Twiss parameters, and emittance, are then retrieved by minimizing the chi-square fit function as defined in [17].

For a comblike beam, the algorithm used to calculate the second momenta of the intensity distribution in the vertical direction is applied to each bunch individually, allowing to determine the emittance for both bunches.

The software for separating the images of the comblike beams was developed in LABVIEW $^{\circledR}$ environment, while the one dedicated to image analysis and emittance calculation in MATLAB ${ }^{\circledR}$. For each of the acquired images the software generates two masks able to separate the regions of interest (ROI) corresponding to the different beams. The masks are created by setting a variable threshold (dynamically set by the program), which neglects all the pixels with intensity less than the threshold. For each iteration the contiguous regions (called particles) exceeding the threshold are calculated. The routine raises the threshold until only two particles remain which serve to identify the ROI containing the two beams (see Fig. 3).

Two subsets are obtained from the measurements, each containing images of a single beam and the information (energy, current) relating to it. The image shown in Fig. 3 refers to a working point developed for two color FEL operation [19], with total charge of $150 \mathrm{pC}$, in a ratio 55\% to $45 \%$, with energies of about 85.7 and $86.2 \mathrm{MeV}$. This train is longitudinally compressed via velocity bunching.

Figure 4 reports the fit for the vertical emittance measurement on both screens, where bunches No. 1 and No. 2 are measured on D1 and the whole beam on U3, showing a perfect agreement between data and theoretical model as reported in Table IV. The whole beam emittance is greater than the emittance of the single pulses due to both a mismatch of the two bunches, as also $\alpha$ and $\beta$ testify, and a centroids misalignment.

TABLE III. Comparison of single bunch emittance measurement on screen U3 and D1.

\begin{tabular}{lclc}
\hline \hline Flag & Emittance (mm-mrad) & \multicolumn{1}{c}{$\alpha$} & $\beta(\mathrm{m})$ \\
\hline U3 & $0.89(0.03)$ & $-1.17(0.08)$ & $13.4(0.7)$ \\
D1 & $0.91(0.07)$ & $-1.4(0.4)$ & $12.3(0.8)$ \\
\hline \hline
\end{tabular}

This measurement was of paramount importance in order to properly match a train of two bunches injected into an undulator with a seed laser to produce two colors seeded FEL radiation [20].

When we consider to measure the horizontal emittance we must take into account also the energy spread. Using again the $\sigma$-matrix formalism we have

$$
\sigma_{11}=R_{11}^{2} \sigma_{011}+2 R_{11} R_{12} \sigma_{012}+R_{12}^{2} \sigma_{022}+R_{16}^{2} \sigma_{066},
$$

where $\sigma_{066}$ is the relative energy spread and $\sigma_{11}=\sqrt{\varepsilon_{x} \beta_{x}}$ is the horizontal dimension on the screen. In this case the emittance can be retrieved only if $R_{16}^{2} \sigma_{066}$ is not the leading term. Otherwise the change in the quadrupole current can

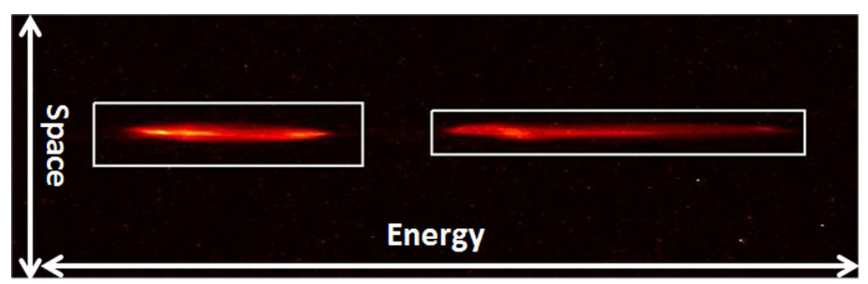

FIG. 3. Emittance measurement in the vertical plane on screen D1.

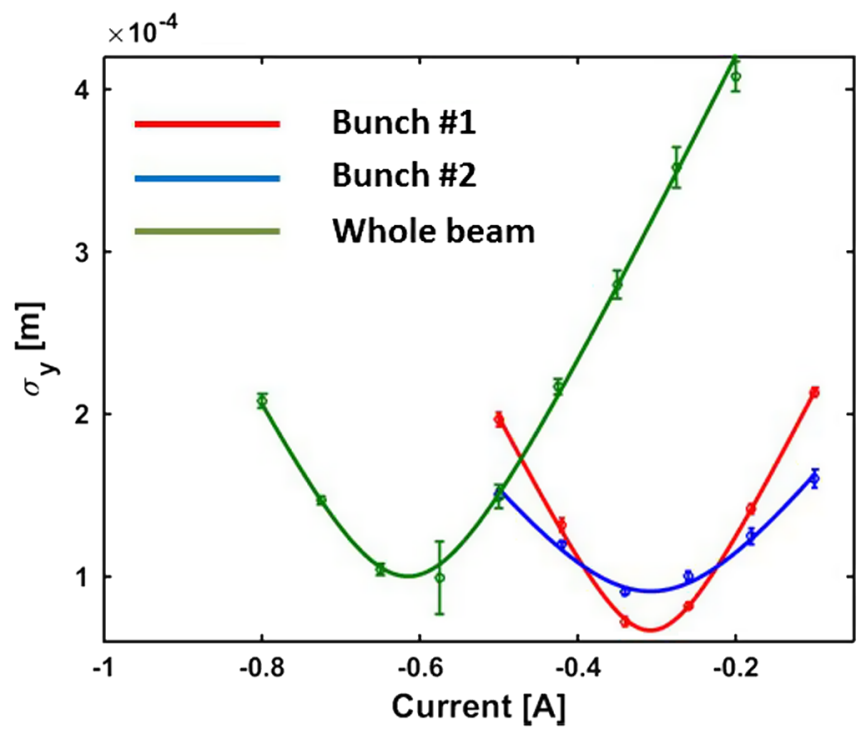

FIG. 4. Emittance measurement in the vertical plane on screen D1 and on screen U3. The single bunches are measured on D1 and the whole on U3. 
TABLE IV. Comparison of single bunch emittance measurement on screen U3 and D1.

\begin{tabular}{lccc}
\hline \hline Bunch & Emittance (mm-mrad) & \multicolumn{1}{c}{$\alpha$} & $\beta(\mathrm{m})$ \\
\hline No. 1 & $1.77(0.13)$ & $-2.1(0.4)$ & $27.2(1.9)$ \\
No. 2 & $1.62(0.12)$ & $-0.9(0.2)$ & $12.3(0.8)$ \\
Whole & $2.31(0.16)$ & $-0.66(0.13)$ & $19.8(1.4)$ \\
\hline \hline
\end{tabular}

have only a very small or even negligible effect. For example in the case of two bunches separated both in time and energy, the energy spread induced by velocity bunching can be on the order of $0.5 \%$ or greater, preventing the use of our system. However when we run on crest, with reduced energy spread we can still use the dipole to measure the horizontal emittance of the single bunch. Also in this case we cross-checked the system measuring in both screens in single bunch operation.

In Fig. 5 is reported a fit of the horizontal size of a single bunch with the previous function. In this way also the energy spread of the beam is evaluated by the fitting routine, being in this case $0.2 \%$. The comparison between measurement on screens D1 and U3 is reported in Table V. Also in this case there is an excellent agreement for the emittance value and $\beta$, while the estimation of the $\alpha$ parameter remains only fair.

\section{B. Bunches mainly separated in time}

The most interesting case for PWFA is represented by a comblike beam separated in time, but not in energy. In this case the emittance measurement is performed only on U3 and the RFD can be used to separate the bunches. A quadrupole scan can be performed assuming that the RFD transport matrix is basically a drift in the plane where there is not deflecting voltage, in our case the horizontal plane.

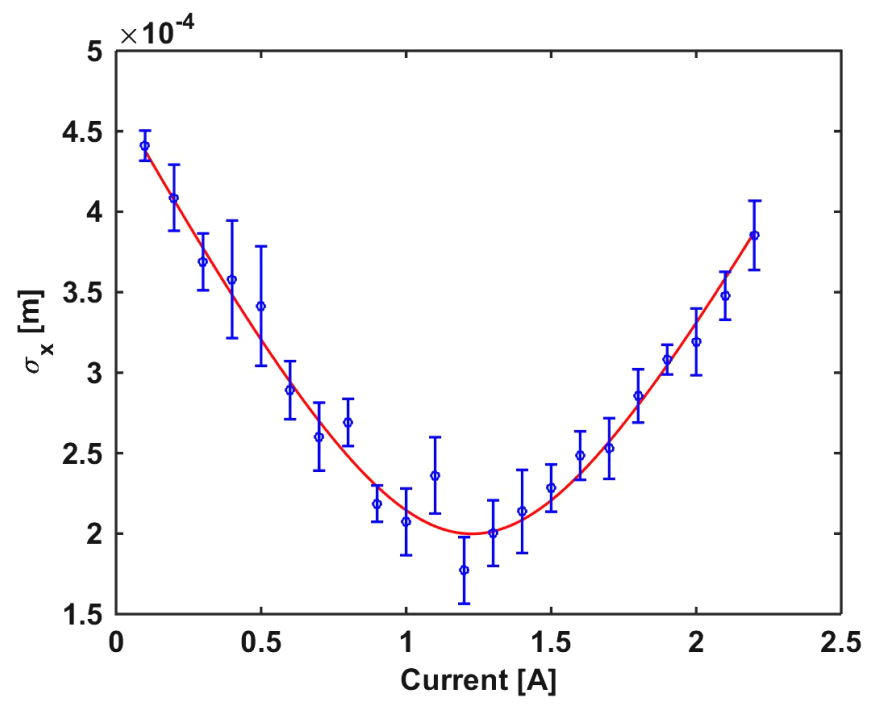

FIG. 5. Emittance measurement in the horizontal plane on screen D1.
TABLE V. Comparison of single bunch emittance measurement on screen U3 and D1.

\begin{tabular}{lclc}
\hline \hline Screen & Emittance (mm-mrad) & \multicolumn{1}{c}{$\alpha$} & $\beta(\mathrm{m})$ \\
\hline D1 & $1.4(0.13)$ & $-1.7(0.4)$ & $19.2(1.3)$ \\
U3 & $1.4(0.10)$ & $-0.94(0.1)$ & $19.0(1.0)$ \\
\hline \hline
\end{tabular}

To check it we compared two quadrupole scans with RFD on and off imaging the beam onto screen U3 as shown in Fig 6.

As expected there is no influence of the RFD, and also the values of the emittance and the Twiss parameters are barely the same. With such a system we measured the horizontal emittance of every single bunch in a train of

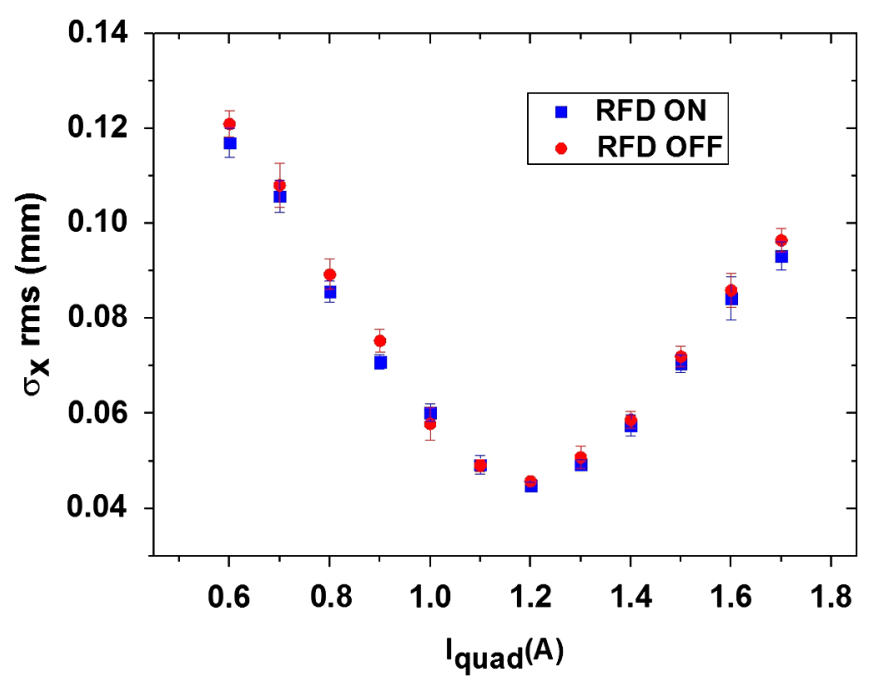

FIG. 6. Quadrupole scan comparison on screen U3 with RFD on and off in the horizontal plane.

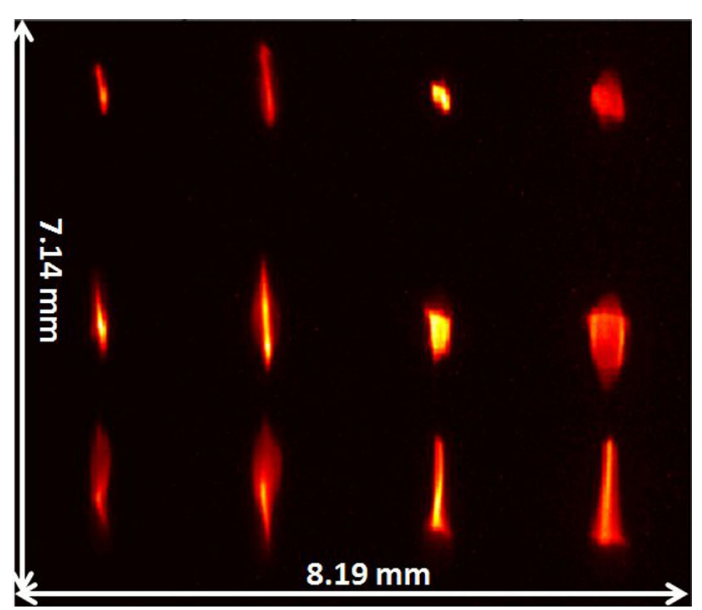

FIG. 7. Train of two drivers and one witness bunch with four different values of quadrupole current. The witness is on the top of the picture. 


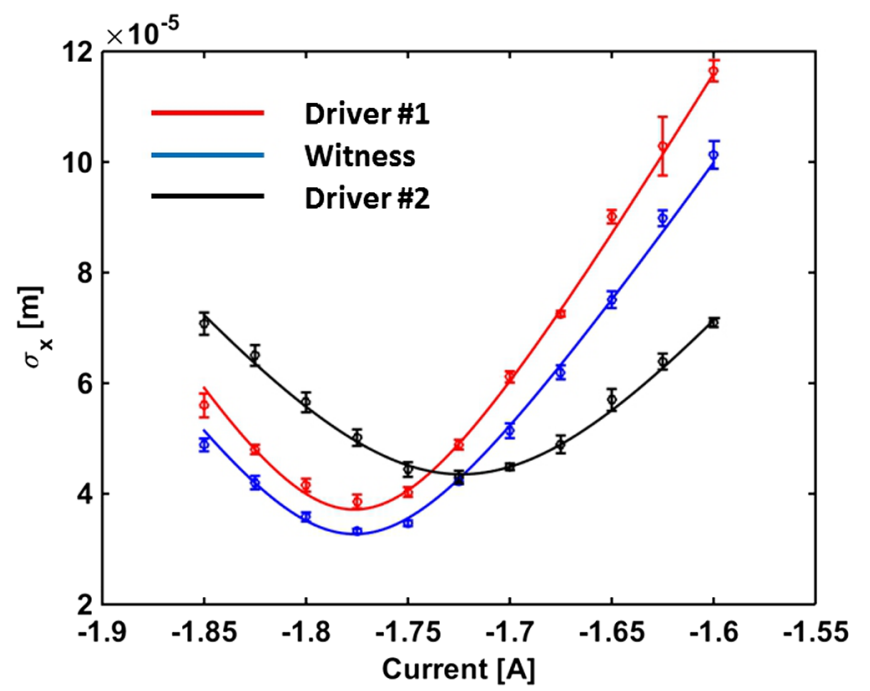

FIG. 8. Quadrupole scan on screen U3 with RFD on, in the horizontal plane. Here are reported the fits for every bunch in the train. It refers to the bunches shown in Fig. 7.

three pulses. In Fig. 7 is reported the superposition of several screenshots showing the behavior of the bunches during the scan.

The total charge was $130 \mathrm{pC}, 20 \%$ of them in the witness and the other equally divided in the two driver beams. The time distance between bunches was 1 ps between drivers and $1.5 \mathrm{ps}$ between the witness and the last driver.

Our software automatically extracts the three regions of interest belonging to every bunch and computes the rms horizontal size for all of them. The result of the quadrupole scan with superimposed fit is shown also in Fig. 8.

The emittance was $1.22(0.06) \mathrm{mm}$-mrad for the witness, $1.38(0.04) \mathrm{mm}$-mrad for driver No. 1, and $1.61(0.07) \mathrm{mm}$ $\mathrm{mrad}$ for driver No. 2. The measured working point is far from being optimized.

\section{CONCLUSIONS}

In this paper we have discussed the problems related with the transverse and longitudinal diagnostics of comblike beams. We have seen that both longitudinal and transverse parameters are fundamental for several different applications. Longitudinal measurements are carried on using a RFD with a dipole, allowing a single shot measurement. Resolution in the order of $35 \mathrm{fs}$ in time and few $\mathrm{keV}$ in energy have been achieved. For the emittance measurement we have divided the case in which the separation is mainly in energy or in time. In the first case we performed a quadrupole scan using the dipole and we found that the vertical emittance can be always measured. For the horizontal emittance it results that there is a limited range of energy spread in which our analysis is valid, until its value is large enough that the variation of the quadrupole strength has not a detectable effect on beam dimension. In the case of beams mainly separated in time, as requested for PWFA for instance, we performed emittance measurement with quadrupole and RFD. We measured the emittance of every single bunch in the direction orthogonal to the deflecting voltage. We always crosschecked all the results of our methods operating in the single bunch regime.

\section{ACKNOWLEDGMENTS}

This work has been partially funded by the EU Commission in the Seventh Framework Program, Grant Agreement No. 312453 EuCARD-2 and partially funded by the Italian Minister of Research in the framework of FIRB-Fondo per gli Investimenti della Ricerca di Base, Project No. RBFR12NK5K.

[1] P. Muggli, V. Yakimenko, M. Babzien, E. K. Kallos, and K. P. Kusche, Phys. Rev. Lett. 101, 054801 (2008).

[2] M. Boscolo, M. Ferrario, C. Vaccarezza, I. Boscolo, F. Castelli, and S. Cialdi, Int. J. Mod. Phys. B 21, 415 (2007).

[3] A. A. Lutman, R. Coffee, Y. Ding, Z. Huang, J. Krzywinski, T. Maxwell, M. Messerschmidt, and H.-D. Nuhn, Phys. Rev. Lett. 110, 134801 (2013).

[4] V. Petrillo, M. Anania, M. Artioli, A. Bacci, M. Bellaveglia, E. Chiadroni, A. Cianchi, F. Ciocci, G. Dattoli, D. Di Giovenale et al., Phys. Rev. Lett. 111, 114802 (2013).

[5] Y. Shen, X. Yang, G. L. Carr, Y. Hidaka, J. B. Murphy, and X. Wang, Phys. Rev. Lett. 107, 204801 (2011).

[6] E. Chiadroni, M. Bellaveglia, P. Calvani, M. Castellano, L. Catani, A. Cianchi, G. Di Pirro, M. Ferrario, G. Gatti, O. Limaj et al., Rev. Sci. Instrum. 84, 022703 (2013).

[7] M. Litos, E. Adli, W. An, C. Clarke, C. Clayton, S. Corde, J. Delahaye, R. England, A. Fisher, J. Frederico et al., Nature (London) 515, 92 (2014).

[8] R. Pompili, A. Cianchi, D. Alesini, M. Anania, A. Bacci, M. Bellaveglia, M. Castellano, E. Chiadroni, D. Di Giovenale, G. Di Pirro et al., Nucl. Instrum. Methods Phys. Res., Sect. A 740, 216 (2014).

[9] M. Ferrario, D. Alesini, M. Anania, A. Bacci, M. Bellaveglia, O. Bogdanov, R. Boni, M. Castellano, E. Chiadroni, A. Cianchi et al., Nucl. Instrum. Methods Phys. Res., Sect. B 309, 183 (2013).

[10] D. Alesini, G. Di Pirro, L. Ficcadenti, A. Mostacci, L. Palumbo, J. Rosenzweig, and C. Vaccarezza, Nucl. Instrum. Methods Phys. Res., Sect. A 568, 488 (2006).

[11] A. Cianchi, D. Alesini, A. Bacci, M. Bellaveglia, R. Boni, M. Boscolo, M. Castellano, L. Catani, E. Chiadroni, S. Cialdi et al., Phys. Rev. ST Accel. Beams 11, 032801 (2008).

[12] P. Emma, J. Frisch, and P. Krejcik, LCLS Technical Note No. 12.

[13] D. Alesini, E. Chiadroni, M. Castellano, L. Cultrera, G. Di Pirro, M. Ferrario, D. Filippetto, G. Gatti, L. Ficcadenti, E. Pace, C. Vaccarezza, C. Vicario, B. Marchetti, A. Cianchi, A. Mostacci, and C. Ronsivalle, in Proceedings of the 9th European Workshop on Beam Diagnostics and Instrumentation for Particle Accelerators 
(DIPAC09), Basel, Switzerland (Paul Scherrer Insitut, Basel, Switzerland, 2009).

[14] M. Ferrario, D. Alesini, A. Bacci, M. Bellaveglia, R. Boni, M. Boscolo, M. Castellano, E. Chiadroni, A. Cianchi, L. Cultrera et al., Phys. Rev. Lett. 104, 054801 (2010).

[15] M. G. Minty and F. Zimmermann, Measurement and Control of Charged Particle Beams (Springer-Verlag, Berlin, 2003).

[16] K. L. Brown and S. K. Howry, Transport/360: A computer program for designing charged particle beam transport systems, Technical Report, Stanford Linear Accelerator Center, California (Stanford University, Stanford, California, 1970).
[17] A. Mostacci, M. Bellaveglia, E. Chiadroni, A. Cianchi, M. Ferrario, D. Filippetto, G. Gatti, and C. Ronsivalle, Phys. Rev. ST Accel. Beams 15, 082802 (2012).

[18] A. Mostacci, A. Bacci, M. Boscolo, E. Chiadroni, A. Cianchi, D. Filippetto, M. Migliorati, P. Musumeci, C. Ronsivalle, and A. Rossi, Rev. Sci. Instrum. 79, 013303 (2008).

[19] C. Ronsivalle, M. Anania, A. Bacci, M. Bellaveglia, E. Chiadroni, A. Cianchi, F. Ciocci, G. Dattoli, D. Di Giovenale, G. Di Pirro et al., New J. Phys. 16, 033018 (2014).

[20] A. Petralia, M. Anania, M. Artioli, A. Bacci, M. Bellaveglia, M. Carpanese, E. Chiadroni, A. Cianchi, F. Ciocci, G. Dattoli et al., Phys. Rev. Lett. 115, 014801 (2015). 